\title{
Series-Parallel Planar Ordered Sets Have Pagenumber Two
}

\author{
(Extended Abstract) \\ Mohammad Alzohairi ${ }^{1} \quad$ Ivan Rival $^{2}$ \\ 1 Department of Mathematics and Statistics \\ University of Ottawa \\ Ottawa K1N 6N5, Canada \\ alzohair@csi.uottawa.ca \\ ${ }^{2}$ Department of Computer Science \\ University of Ottawa \\ Ottawa K1N 6N5 Canada \\ rival@csi.uottawa.ca
}

\begin{abstract}
The pagenumber of a series-parallel planar $P$ is at most two. We present an $O\left(n^{3}\right)$ algorithm to construct a two-page embedding in the case that it is a lattice. One consequence of independent interest, is a characterization of series-parallel planar ordered sets.
\end{abstract}

\section{Introduction}

A book embedding of a graph $G$ consists of an embedding of its nodes along the spine of a book (i.e., a linear ordering of the nodes), and an embedding of its edges on pages so that edges embedded on the same page do not intersect. In a book embedding for an ordered set $P$ the vertices of $P$ on the spine form a linear extension (a total order $L=\left\{x_{1}<x_{2}<\cdots<x_{n}\right\}$ of the elements of $P$ is a linear extension if $x<y$ in $L$ whenever $x<y$ in $P$ ).

We say a covers $b$ (or $b$ covered $b y a$ ) in the ordered set $P$, and write $a \succ b$ (or $b \prec a$ ), if whenever $a>c \geq b$ then $c=b$. Also, we say $a$ is an upper cover of $b$, or $b$ is a lower cover of $a$, or $(a, b)$ is an edge in $P$. We say $a$ is a minimal (respectively, maximal) element of $P$ if $a$ has no lower covers (respectively, $a$ has no upper covers). We denote the set of all minimals (respectively, maximals) of $P, \min (P)$ (respectively, $\max (P))$. The covering graph of $P, \operatorname{cov}(P)$, is the graph whose vertices are the elements of $P$, and the pair $\{a, b\}$ forms an edge in $\operatorname{cov}(P)$ if $a \succ b$ or $a \prec b$. It is possible to orient $\operatorname{cov}(P)$ in such a way the $y$-coordinate of $a$ is less than the $y$-coordinate of $b$ if $a \prec b$ and the edge $(a, b)$ 
does not pass through any other element of $P$. We call such drawing an upward drawing of $P$.

The pagenumber in both cases (page $(G)$, respectively page $(P))$ is the minimum number of pages needed taken over all linear layouts for graphs and all linear extensions for an ordered set. For instance, page $(P)=2$ for the ordered set illustrated in Figure 1, while page $(\operatorname{cov}(P))=1$. On the other hand the planar lattice in Figure 2 required three pages (this example is due to J. Czyzowicz $[7])$.

The pagenumber was first defined for graphs by Bernhart and Kainen [1], who conjectured that planar graphs may require an arbitrary large number of pages. In a series of attempts, it was finally established by Yannakakis [11], that page $(G) \leq 4$ for every planar graph $G$, and this upper bound is achieved. Fraysseix, Mendez and Pach [4] have shown that the pagenumber of any planar graph with quadrilateral faces is at most two.

The page number for ordered sets has been introduced by Nowakowski and Parker [7], who show that page $(P)=1$ if and only if $\operatorname{cov}(P)$ is a forest. Also, they derive a general lower bound on the page number of ordered sets and upper bounds for special classes of ordered sets. Hung [3] shows that there exists a 48-element planar ordered set which needs four pages (see Figure 3). Moreover, no planar ordered set with pagenumber five is known. Sysło [9] provides a lower bound on the page number in terms of its bump number. He also shows that, page $(P) \leq 2$ if the jump number of $P$ is one. Ordered sets with jump number two can have an arbitrarily large page number. Later, Heath and Pemmaraju [8] gave a sequence of ordered sets each with planar covering graph and with unbounded page number. Computationally, we recently proved that finding the minimum number of pages required for a fixed linear extension of an ordered set is NP-complete.

In section 2 we study the structure of series-parallel planar lattices. In section 3 we will construct, for a series-parallel planar lattice $P$, an $O\left(n^{3}\right)$ two-page algorithm where $n$ is the number of the elements of $P$. In section 4 we continue the study of the structure of series-parallel planar ordered sets. In section 5 we exploit the fact that the completion $\bar{P}$ of a series-parallel planar ordered set $P$ is itself a series-parallel planar lattice. We use the result in section 3 to obtain a two-page linear extension $\bar{L}$ of $\bar{P}$, which we transfer to a two-page linear extension of $P$. In section 6 we give three open problems related to the pagenumber problem.

\section{Structure of series-parallel planar lattices}

The linear sum $P \oplus Q$ of the two disjoint ordered set $P, Q$ is an ordered set on $P \cup Q$, that is, $a \leq b$ if
1. $a \leq b$ in $P$, or
2. $a \leq b$ in $Q$, or
3. $a \in P$ and $b \in Q$.

If we eliminate the third condition of the definition of linear sum, we will have the disjoint sum $P+Q$ of $P, Q$. 
An ordered set $P$ is series-parallel if $P$ can be constructed from singletons using only the constructions of disjoint sum + and linear sum $\oplus$. In other words, $P$ can be decomposed into singletons using only disjoint sum and linear sum. For instance, the the series-parallel lattice illustrated in Figure 4 can be decomposed into

$$
1 \oplus(((2+6) \oplus 3 \oplus(4+(7 \oplus(8+(10 \oplus 11)+12+13) \oplus 9)))+(14 \oplus(15+17) \oplus 16)) \oplus 5
$$

For $a \neq b$ in the ordered set $P$ we say $a$ is comparable to $b$ if either $a<b$ or $a>b$. Otherwise, $a$ is noncomparable to $b$, write $a \| b$. An antichain is a subset $A$ of an ordered set $P$ such that any two distinct elements of $A$ are noncomparable. Dually, a chain of $P$ is a subset $C$ of $P$ where, each pair of $C$ are comparable.

A four-element subset $\{a, b, c, d\}$ of an ordered set $P$ forms an $\mathbf{N}$ if the only comparabilities among them in $P$ are $a<c, b<c$ and $b<d$. It is known that an ordered set is a series-parallel if and only if it contains no such $\mathbf{N}$ [10].

Fix a planar embedding of $P$, and let $C=\left\{x_{1}<x_{2}<\ldots<x_{n}\right\}$ be the left boundary chain. For each $x \in P-C$ define the interval $I(x)=\left(x_{i}, x_{j}\right)$, where

$$
x_{i}=\max _{1 \leq k<n}\left\{x>x_{k}\right\} \quad x_{j}=\min _{1<k \leq n}\left\{x<x_{k}\right\}
$$

Of course, $j \geq i+1$. Notice that, $j>i+1$ because if $j=i+1$ then the edge $\left(x_{i+1}, x_{i}\right)$ will not be an essential edge. (An edge $(a, b)$ is not essential if there is $c$ such that $a<c<b$.)

Notice that, every pair of these intervals is either disjoint or one contains the other. Hence the set of intervals ordered by inclusion is a forest (an ordered set $P$ is a forest if the graph $\operatorname{cov}(P)$ is a forest). For $y, z \in P-C$, say $y \sim z$ if $I(y)=I(z)$. It is clear that this relation is an equivalence relation. Call the equivalence classes components.

For example, the components of the series-parallel order in Figure 4 are: $C_{1}=\{7,8,9,10,11,12,13\}$ which corresponds to the interval $(3,5)$;

$C_{2}=\{6\}$ which corresponds to the interval $(1,3)$; $C_{3}=\{14,15,16,17\}$ which corresponds to the interval $(1,5)$.

The forest obtained by ordering the intervals by inclusion is shown in Figure 6 . We can show that there are no edges between the components.

Here are a few elementary terms. Fix a lattice $P$ and fix a planar upward drawing of it. For noncomparable element $a, b \in P$ such that $a \succ c$ and $b \succ c$, we say $a$ is left of $b$ if any horizontal segment (moving from left to right) which cuts both edges, always cuts the edge $(c, a)$ before the edge $(c, b)$. For arbitrary noncomparable elements $a$ and $b(a \| b)$ in $P$ say that $a$ is left of $b$, denoted $a \lambda b$, if $a^{\prime}$ is left of $b^{\prime}$, where $a \geq a^{\prime} \succ \inf (\{a, b\})$ and $b \geq b^{\prime} \succ \inf (\{a, b\})$. An element $a$, which does not belong to the maximal chain $C$ is left of $C$ if there is $b \in C$ such that $a \lambda b$. In fact, $a$ is left of $b$ if $a$ is left to any maximal chain containing $b$. (Of course, all of these ideas are ambidextrous. If $a$ is left of $b$ then $b$ is right of $a$, etc.)(For details see [5].)

Once equipped with the equality relation, $\lambda$ becomes an order relation on $P$, denoted $P_{\lambda}$. (This result is due to J. Zilber see [2] page 32, ex. 7(c).) For 
example, the ordered set in Figure 5 is $P_{\lambda}$ where $P$ is the planar lattice in Figure 4.
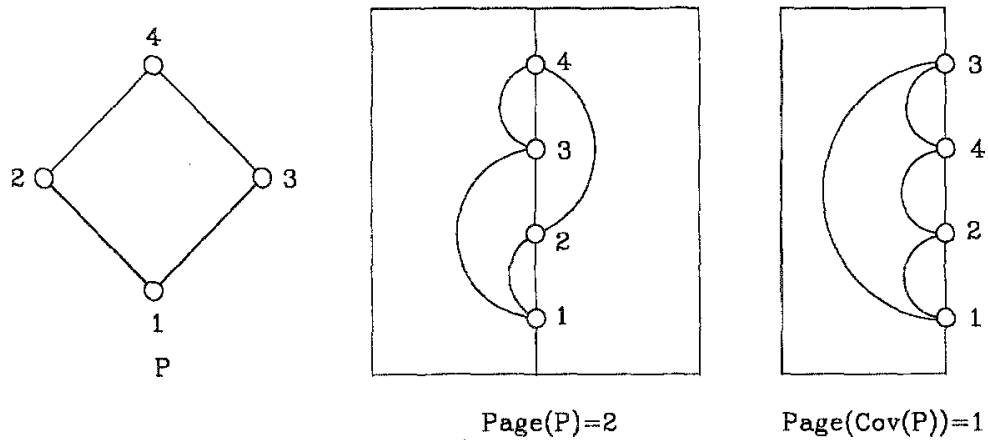

Figure 1

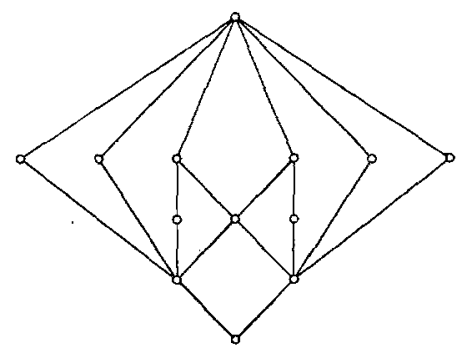

Figure 2

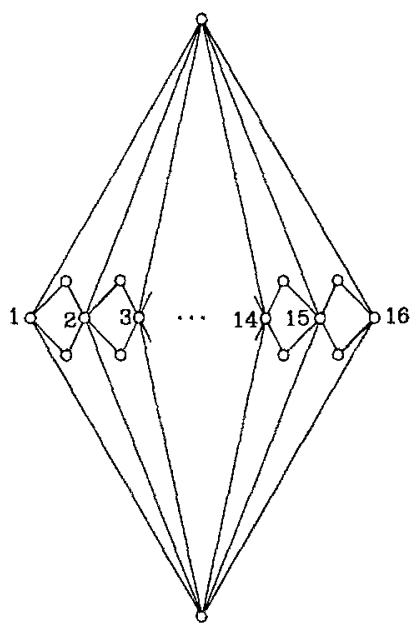

Figure 3

For a series-parallel planar lattice $P$, fix a planar upward drawing of $P$, and define the sequence of peels of $P$ as follows:

$L_{0}=\{x \in P: x$ belongs to the left boundary $\}$.

$L_{1}=\left\{x \in P-L_{0}:\right.$ if $y$ lies to the left of $x$, then $\left.y \in L_{0}\right\}$.

$L_{2}=\left\{x \in P-\left(L_{0} \cup L_{1}\right):\right.$ if $y$ lies to the left of $x$, then $\left.y \in L_{0} \cup L_{1}\right\}$.

$L_{t}=\left\{x \in P-\left(L_{0} \cup L_{1} \cup \cdots \cup L_{t-1}\right):\right.$ if $y$ lies to the left of $x$, then $y \in L_{0} \cup$ $\left.L_{1} \cup \cdots \cup L_{t-1}\right\}$.

We call any $L_{i}$ a peel of $P$. Actually, the peels of a planar lattice $P$ are the levels of $P_{\lambda}$, where $P_{\lambda}$ the underlying set $P$ ordered by $\lambda$. 


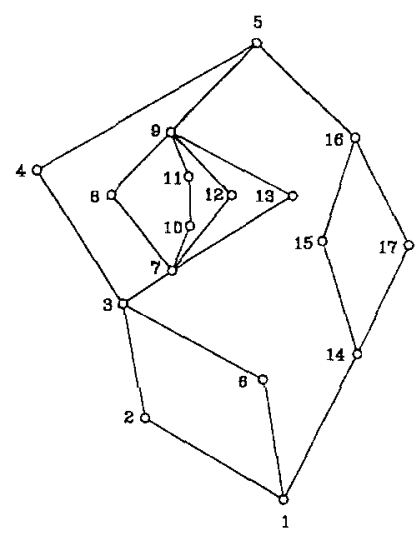

Figure 4

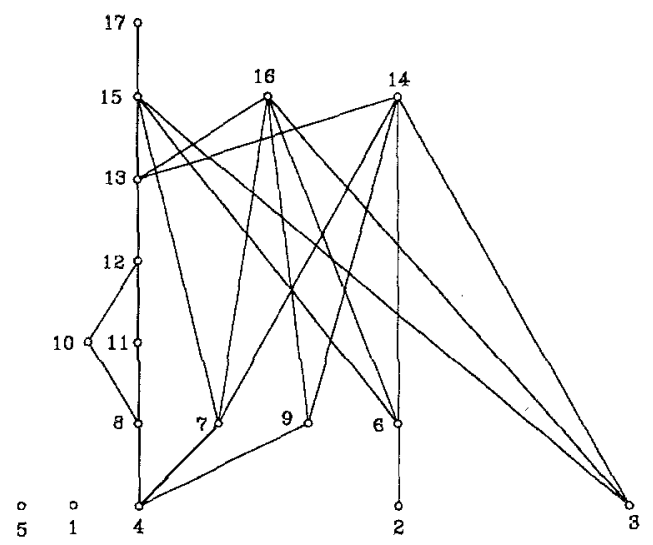

Figure 5

Thus, $L_{i}=\min \left(P_{\lambda}-\left(\bigcup_{j=0}^{i-1} L_{j}, 0 \leq j \leq t\right)\right.$

Of course, $t$ is equal the height of $P_{\lambda}$, where the height of an ordered set is less one than the maximum number of elements of a chain.

For example, in the series-parallel ordered set $P$ with respect to the upward drawing shown in Figure 4
$L_{0}=\{1,2,3,4,5\}$
$L_{1}=\{6,7,8,9\}$
$L_{2}=\{10,11\}$
$L_{3}=\{12\}$
$L_{4}=\{13\}$
$L_{5}=\{14,15,16\}$
$L_{6}=\{17\}$

Lemma 1 Let $P$ be a series-parallel planar lattice. If $0 \leq i \leq t$, then

1. for any $x \in L_{i}, i>0$, there exist $y \in L_{i-1}$ such that $x$ lies to the right of $y$,

2. the peel $L_{i}$ forms a chain,

3. the number of peels equals width $(P)-1$ (width $(P)$ is the maximum size of antichain in $P$ ).

Call a chain $C$ in $P$ is saturated if all of its covering relations, are covering relations in $P$. Each chain decomposes into its (maximal) saturated chains.

In a series-parallel planar lattice $P$ each peel $L_{i}$ can be decomposed into maximal saturated subchains $C_{i 1}, C_{i 2}, \ldots, C_{i n_{i}}$ called the clamped chains for $P$.

For a clamped chain $C_{i j} \in L_{i}, i \geq 1$ define:

$$
\begin{aligned}
& l\left(C_{i j}\right)=\left\{y \in L_{0} \cup L_{1} \cup \cdots \cup L_{i-1}: \inf \left(C_{i j}\right) \succ y\right\} \\
& u\left(C_{i j}\right)=\left\{y \in L_{0} \cup L_{1} \cup \cdots \cup L_{i-1}: \sup \left(C_{i j}\right) \prec y\right\}
\end{aligned}
$$

For example the table below shows the clamped chains in the series parallel planar lattice in Figure 4. 


\begin{tabular}{|l|c|c|}
\hline Clamped chain $C_{i j}$ & $l\left(C_{i j}\right)$ & $u\left(C_{i j}\right)$ \\
\hline$C_{0}=\{1,2,3,4,5\}$ & - & - \\
\hline$C_{11}=\{6\}$ & 1 & 3 \\
\hline$C_{12}=\{7,8,9\}$ & 3 & 5 \\
\hline$C_{21}=\{10,11\}$ & 7 & 9 \\
\hline$C_{31}=\{12\}$ & 7 & 11 \\
\hline$C_{41}=\{13\}$ & 7 & 9 \\
\hline$C_{51}=\{14,15,16\}$ & 1 & 5 \\
\hline$C_{61}=\{17\}$ & 14 & 16 \\
\hline
\end{tabular}

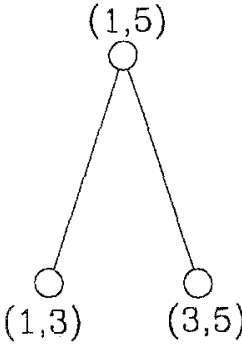

Figure 6

Lemma 2 Let $C_{i j}$ be a clamped chain in a series-parallel planar lattice $P$.

1. Each $l\left(C_{i j}\right)$ and $u\left(C_{i j}\right)$ is unique.

2. Each $x \in C_{i j}-\left\{\right.$ inf $\left.C_{i j}, s u p C_{i j}\right\}$ has neither lower covers nor upper covers in $L_{0} \cup L_{1} \cup \cdots \cup L_{i-1}$. Also, if inf $C_{i j} \neq s u p C_{i j}$ then inf $C_{i j}$ (respectively, $\sup \left(C_{i j}\right)$ ) has no lower (respectively, upper) covers in $L_{0} \cup L_{1} \cup \cdots \cup L_{i-1}$,

3. If $u\left(C_{i j}\right) \in C_{k m}$, then $l\left(C_{i j}\right) \in C_{k m} \cup\left\{l\left(C_{k m}\right)\right\}$.

4. inf $C_{i j}$ (respectively, sup $C_{i j}$ ) has a unique lower (respectively, upper) cover in $P$.

\section{Two pages are enough}

In this section we will give an $O\left(n^{3}\right)$ two-page algorithm for a series-parallel planar lattice $P$, where $n$ is the number of elements of $P$.

To obtain a two-page linear extension of a series-parallel planar lattice $P$

(i) Fix a planar upward drawing for $P$.

(ii) List the clamped chains of $P$ in the following order $C_{0}, C_{11}, C_{12}, \ldots, C_{1 n_{1}}, C_{21}, C_{22}, \ldots, C_{2 n_{2}}, \ldots, C_{w 1}, C_{22}, \ldots, C_{w n_{1 w}}$. We will process chain by chain according to the above order.

(iii) Put $C_{0}$ on the spine of the book. Draw the bottom edge on the right page and draw all other edges on the left page.

(iv) Suppose two pages are enough up to $C_{i j-1}$. For $C_{i j}$ put all the elements of $C_{i j}$ right below $u\left(C_{i j}\right)$. Draw the edge $\left(\right.$ inf $\left.f\left(C_{i j}\right), l\left(C_{i j}\right)\right)$ on the right page and draw all $C_{i j}$ edges and the edge $\left(u\left(C_{i j}\right), \sup \left(C_{i j}\right)\right)$ on the left page.

Call this algorithm the two-page algorithm.

Figure 7, illustrates the steps of the two-page algorithm applied on the seriesparallel planar lattice $P$ in Figure 4.

A greedy linear extension of an ordered set $P$ is a linear extension $x_{1}<$ $x_{2}<\cdots<x_{n}$ of $P$ such that $x_{1} \in \min (P)$ and, for $i \geq 1, x_{i+1} \in \min (P-$ $\left.\left\{x_{1}, x_{2}, \cdots, x_{i}\right\}\right)$ and, if possible, $x_{j+1}>x_{j}$. Thus, a greedy linear extension obtained by following "the rule climb as heigh as you can". 
A left greedy linear extension of $P$ is that greedy linear extension whose the $i$ th element $x_{i+1}$ is the (unique) left-most element belonging to $\min (P$ $\left.\left\{x_{1}, x_{2}, \cdots, x_{i}\right\}\right)$
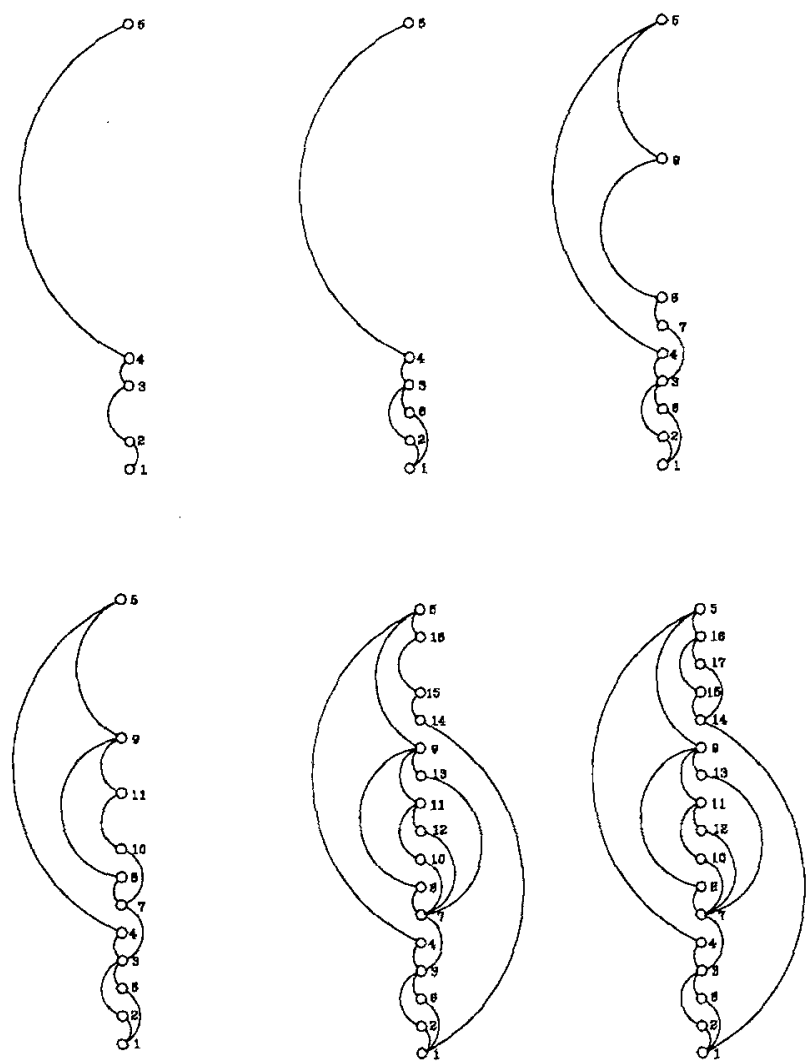

Figure 7

Lemma 3 Let $P$ be a series-parallel planar lattice. If $L$ is the permutation obtained by the two-page algorithm, then

1. $L$ is a linear extension of $P$,

2.if $x \| y$ in $P$, and $y$ lies to the left of $x$, then $y<x$ in L. (i.e., $L$ is a left greedy linear extension).

Theorem 4 The two-page algorithm for an n-element series-parallel planar lattice produces a two-page linear extension $L$ in $O\left(n^{3}\right)$ time.

For the complexity, we can find the peel $C_{0}$ by checking for each $x \in P$ if there is $y \in P-\{x\}$ such that $y \| x$ and $y$ lies to the left of $x$. Thus, we need at most $n^{3}$ comparison operations to obtain the peels of $P$. To obtain the clamped chains of a certain peel we need first to sort it in $O(n \log n)$ comparisons, then determine the covering relations in this peel and that can be done in $O(n-1)$ comparisons. 
Therefore, we can find all clamped chains in $O\left(n^{2} \log n\right)$ comparisons. For each clamped chain $C_{i j}$ we can find $u\left(C_{i j}\right)$ by find the element in $L_{i-1}$ which covers in $f\left(C_{i j}\right)$ and this can be done in $O(n)$ comparisons. Thus, we can find $u\left(C_{i j}\right)$ and $l\left(C_{i j}\right)$ for all clamped chains $C_{i j}$ in $O\left(n^{2}\right)$ comparisons. For the distribution of the edges among the two pages we process each edge just one time; thus, we can decide the page for each edge in $O\left(n^{2}\right)$ comparisons. Thus, the whole algorithm can be done in $O\left(n^{3}\right)$ comparisons.

\section{Structure of series-parallel planar ordered sets}

The completion of an ordered set $P$ is the smallest lattice $\bar{P}$ contains $P$ as suborder. Notice that $\bar{P}$ exists and called MacNeille completion (cf. [6].)

First, we will show that the completion $\bar{P}$ of series-parallel ordered set is series-parallel planar lattice.

The question may arise now whether we can transfer the two-page linear extension $\bar{L}$ of $\bar{P}$ (obtained by Theorem 4) to a two-page linear extension $L$ of $P$ ?

For example, we consider the series-parallel planar ordered set $P$ and its completion $\bar{P}$ in Figure 8. In Figure $9, \bar{L}$ is the two-page linear extension of $\bar{P}$ obtained by the two-page algorithm for series-parallel planar lattice. Let $L$ be the linear extension obtained from $\bar{L}$ by removing the elements in $\bar{P}-P$. Notice that, the linear extension $L$ needs at least three pages.

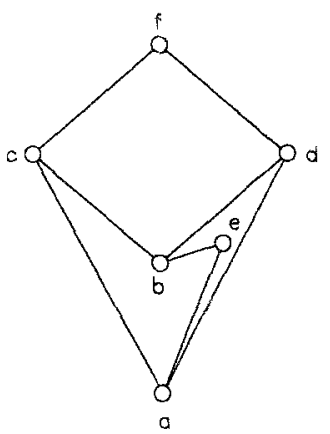

Figure 8

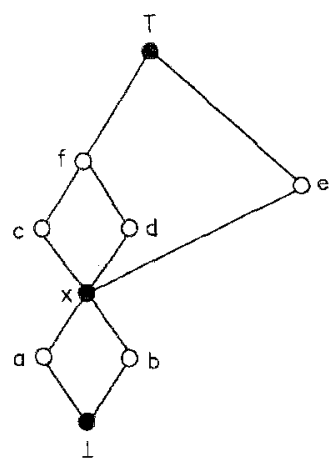

Figure 9

But if we redraw $\bar{P}$ in a different planar embedding as it is in Figure 12, then using the two-page algorithm for series-parallel lattices we will obtain the two-page embedding $\bar{L}$ as it illstrates in Figure 10. In Figure 10, we also, see that the linear extension $L$ of $P$ induced by $\bar{L}$ is a two-page linear extension.

This leads us to this question, whether we can always find a planar embedding of the completion $\bar{P}$ of the series-parallel planar ordered which can lead finally to a two-page linear extension of the ordered set? The answer is yes. 
Lemma 5 If $P$ is a series-parallel planar ordered set and $\bar{P}$ its completion then,
(i) $\bar{P}$ is series-parallel,
(ii) $\bar{P}$ is a planar lattice.

We say the ordered set $P$ contains $K_{m, n}, m, n \geq 2$ if it contains a subset $\left\{a_{1}, a_{2}, \cdots, a_{m}, b_{1}, b_{2}, \cdots, b_{n}\right\}$ satisfying $a_{i} \prec b_{j}$ for $i=1,2, \ldots, m$ and $j=$ $1,2, \ldots, n$. (See Figure 11). Notice that, if $P$ contains $K_{m, n}, m, n \geq 2$ then, the sets $\left\{a_{1}, a_{2}, \cdots, a_{m}\right\}$ and $\left\{b_{1}, b_{2}, \cdots, b_{n}\right\}$ are antichins.

We say $K_{m, n}=\left\{a_{1}, a_{2}, \cdots, a_{m}, b_{1}, b_{2}, \cdots, b_{n}\right\}$ is maximal in $P$ if there is neither $a_{m+1} \neq a_{i}, 1 \leq i \leq m$ satisfying $a_{m+1} \prec b_{j}$ for every $1 \leq j \leq n$, nor $b_{n+1} \neq b_{j}, 1 \leq j \leq n$ satisfying $a_{i} \prec b_{n+1}$ for every $1 \leq i \leq m$. If a planar ordered set contains $K_{m, n}, m, n \geq 2$, then either $m=2$ or $n=2$.

After series of Lemma's, we conclude that if the series-parallel planar ordered set $P$ is not a lattice, then the only obstacle to be a lattice (except the top and the bottom) is existing the maximals of $K_{2, n}$ and/or $K_{m, 2}, m, n \geq 2$.

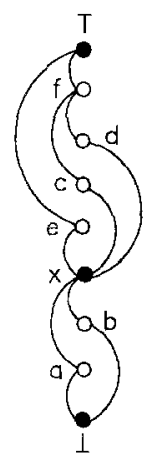

$\overline{\mathrm{L}}$

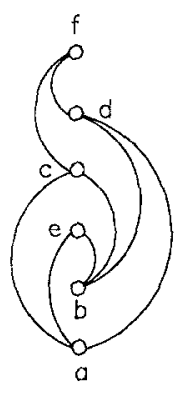

L

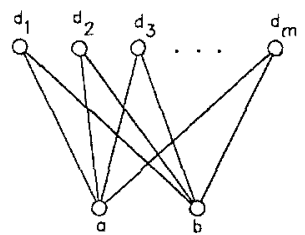

Figure $11 K_{2, m}$

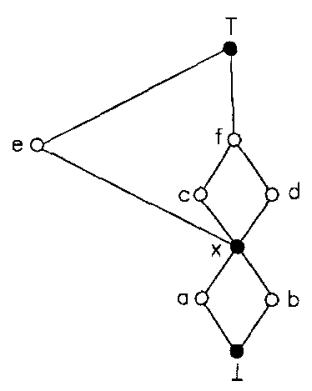

Figure 12

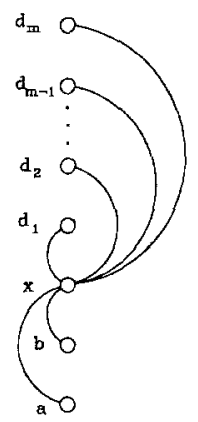

Figure 13

Lemma 6 If the ordered set $P$ contains $K_{2, m}=\left\{a, b, d_{1}, \ldots, d_{m}\right\}, m \geq 2$, and if $P$ satisfies one of the following conditions, then $P$ is not planar.

i) There is an upper bound of some three-element subset of $\left\{d_{1}, \ldots, d_{m}\right\}$. 
ii) $a$ and $b$ have a common lower bound and some two-element subset of $\left\{d_{1}, \ldots, d_{m}\right\}$ has a common upper bound.

iii) There are two different two-element subsets of $\left\{d_{1}, \ldots, d_{m}\right\}$ each of which has an upper bound.

As we indicate in the beginning of this section, obtaining the two-page linear extension for $P$ depends on the planar embedding of the completion $\bar{P}$ of $P$. The next lemma describe such planar embedding.

Lemma 7 Let $P$ be a series-parallel planar ordered set. For each maximal $K_{2, m}=\left\{a, b, d_{1}, \cdots, d_{m}\right\}, m \geq 3$ and each maximal $K_{n, 2}=\left\{d_{1}^{\prime}, \cdot, d_{n}^{\prime}\right\}, n \geq 3$ such that $\left\{d_{1}, d_{2}\right\}$ has a minimal upper bound $d$ and $\left\{d_{1}^{\prime}, d_{2}^{\prime}\right\}$ has a maximal lower bound $d^{\prime}$, there is a planar upward drawing of the completion lattice $\bar{P}$ of $P$ in which $d$ lies to the right of $d_{3}, d_{4}, \cdots, d_{m}$ and $d^{\prime}$ lies to the left of $d_{3}^{\prime}, d_{4}^{\prime}, \cdots, d_{n}^{\prime}$.

\section{The Main result}

In this section we will prove our main result. We will first prove that two pages are enough for a series-parallel planar ordered set. As a consequence, we will give a characterization of series-parallel planar ordered sets.

Theorem 8 If $P$ is series-parallel planar ordered set then, page $(P) \leq 2$.

The transformation algorithm Let $\bar{P}$ be the completion of $P$. By Lemma 5 , $\bar{P}$ is series-parallel planar lattice. Fix a planar embedding of $\bar{P}$ satisfying

1. Whenever $P$ contains a maximal $K_{2, m}=\left\{a, b, d_{1}, \ldots, d_{m}\right\}, m \geq 3$, such that $d$ is an upper bound of $\left\{d_{m-1}, d_{m}\right\}$ then, $d$ lies to the right of $\left\{d_{3}, \ldots, d_{m}\right\}$.

2. Whenever $P$ contains a maximal $K_{m, 2}=\left\{d_{1}, \ldots, d_{m}, a, b\right\}, m \geq 3$, such that $d$ is a lower bound of $\left\{d_{1}, d_{2}\right\}$ then $d$ lies to the left of $\left\{d_{3}, \ldots, d_{m}\right\}$.

This is possible according to Lemma 7 . If $P$ contains either a maximal $K_{2, m}$ or a maximal $K_{m, 2}, m \geq 2$, we may assume that $a$ lies to the left of $b$ and $d_{i}$ lies to the left of $d_{i+1}$ for $1 \leq i \leq m-1$, in $\bar{P}$.

Notice that, if $P$ contains a maximal $K_{2, m}, m \geq 2$, then the set of the upper covers of $a$ is $\left\{d_{1}, \ldots, d_{m}\right\}$ which also is the set of the upper covers of $b$. Also, the set of the lower covers of $d_{i}$ is $\{a, b\}$ for each $i=1, \ldots, m$. Dually for $K_{m, 2}$.

Since $\bar{P}$ is a series-parallel parallel planar lattice, by Theorem 4 there exists a two-page linear extension $\bar{L}$ of $\bar{P}$. We will transfer it to a two-page linear extension $L$ for $P$.

For a four-cycle $C=\{a<c>b<d>a\}$ in an ordered set, a splitting element $x$ satisfying $a, b \leq x \leq c, d$.

If $P$ contains a maximal $K_{2, m}=\left\{a, b, d_{1}, \ldots, d_{m}\right\}, m \geq 2$, such that $x$ is the splitting element of $K_{2, m}$ in $\bar{P}$, then we have $a<b<x<d_{1}<d_{2}<\ldots<$ $d_{m}$ in $\bar{L}$ and the edges distributed as in Figure 13.

Also, if $P$ has a maximal $K_{m, 2}=\left\{d_{1}, \ldots, d_{m}, a, b\right\}, m \geq 2$ such that $x$ is the splitting element of $K_{m, 2}$ in $\bar{P}$, then we have $d_{1}<d_{2}<\ldots<d_{m}<x<a<$ $b$ in $\bar{L}$ and the edges distributed as in Figure 14. 
Since $P$ is planar, by Lemma 6 , if $\{a, b\}$ has a lower (respectively, an upper) bound of $K_{2, m}$ (respectively, $K_{m, 2}$ ) in $P$, then there is no subset of two elements or more of the set $\left\{d_{1}, \ldots, d_{m}\right\}$ which has an upper (respectively, a lower) bound.

To obtain a two-page linear extension $L$ of $P$ from $\bar{L}$

1. Remove the set $\bar{P}-P$ from $\bar{L}$ and all edges connected to its vertcies.

2. For each maximal $K_{2, m}=\left\{a, b, d_{1}, \ldots, d_{m}\right\}, m \geq 2$, in $P$

i) If $\{a, b\}$ has a lower bound in $P$ draw the edges $\left(a, d_{i}\right)$ on the left page and the edges $\left(b, d_{i}\right)$ on the right page (see Figure 15).

ii) If $\left\{d_{m-1}, d_{m-1}\right\}$ has an upper bound in $P$ draw the edges $\left\{\left(b, d_{1}\right),\left(a, d_{i}\right)\right.$ : $1 \leq i \leq m-1\}$ on the left page and draw the edges $\left\{\left(a, d_{m}\right),\left(b, d_{i}\right): 2 \leq\right.$ $i \leq m\}$ on the right page for each $1 \leq i \leq m$ (see Figure 16).

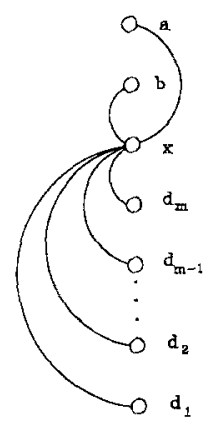

Figure 14

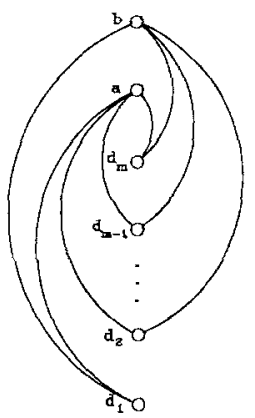

Figure 17

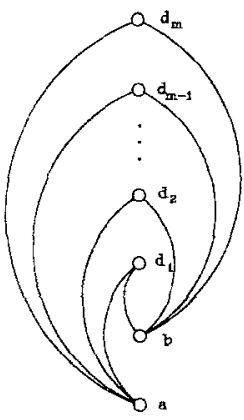

Figure 15

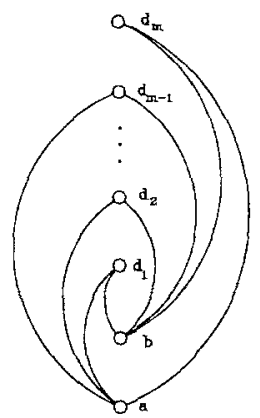

Figure 16

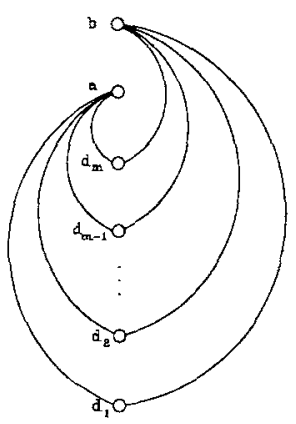

Figure 18

3. For each maximal $K_{m, 2}=\left\{d_{1}, \ldots, d_{m}, a, b\right\}, m \geq 2$, in $P$

i) If $\left\{d_{1}, d_{2}\right\}$ has a lower bound in $P$ draw the edges $\left\{\left(d_{1}, b\right),\left(d_{i}, a\right): 1 \leq i \leq\right.$ $m-1\}$ on the left page and draw the edges $\left\{\left(d_{m}, a\right),\left(d_{i}, b\right): 2 \leq i \leq m\right\}$ on the right page (see Figure 17).

ii) If $\{a, b\}$ has an upper bound in $P$ draw the edges $\left\{\left(d_{i}, a\right): 1 \leq i \leq m\right\}$ on the left page and the edges $\left\{\left(d_{i}, b\right): 1 \leq i \leq m\right\}$ on the right page (see Figure 18). 

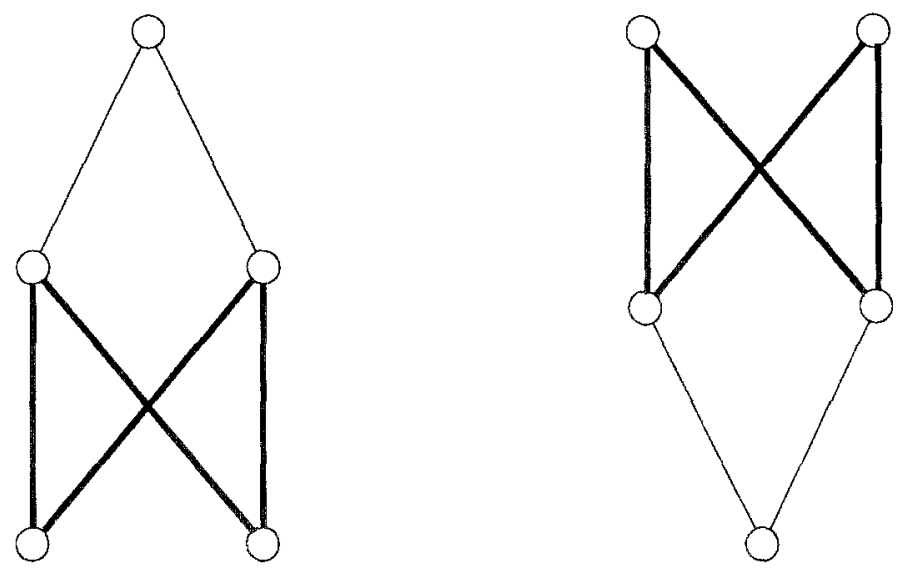

Figure 19 Simple castles.

By Lemma 3, $L$ is greedy linear extension of $P$. We will show that adding the edges of the maximals $K_{2, m}$ and $K_{m, 2}, m \geq 2$, do not create crossing in the same page first for $K_{2, m}$ then for $K_{m, 2}$.

A simple castle is a covering four-cycle with the top or bottom. (The top, or bottom, need not be in a cover relation with the covering four-cycle.)(See Figure 19) A castle is any union of simple castles, which preserves the covering relations of each simple castle. An ordered set $P$ contains a castle $C$ if $C$ is a subset of $P$ and $P$ preserves the covering relations of its simple castles.(See Figure 20)

Corollary 9 Let $P$ be a series-parallel planar ordered set. Then $P$ is planar if and only $P$ contains no $K_{3,3}$ and $P$ contains no nonplanar castle.

Figure 21 illustrates nonplanar ordered sets each of which contains neither $K_{3,3}$ nor a nonplanar castle. In fact, non is series-parallel.

\section{Open problems}

1. Is the pagenumber for planar ordered sets bounded?

This question was first asked by Nowakowski and Parker [7]. Hung [3] gave a 48-element planar ordered set which requires four pages (see Figure 44). No planar ordered set required five pages is known.

2. We proved that two pages are enough to embed a series-parallel planar ordered set. Series-parallel ordered sets have dimension two. Is there a positive integer $k$, such that page $(P) \leq k$, for each planar ordered set of 


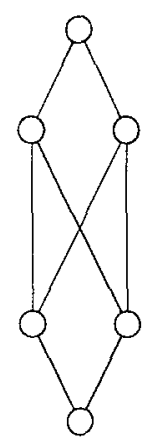

(a)

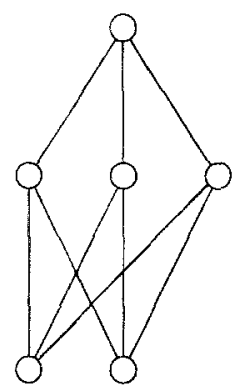

(b)

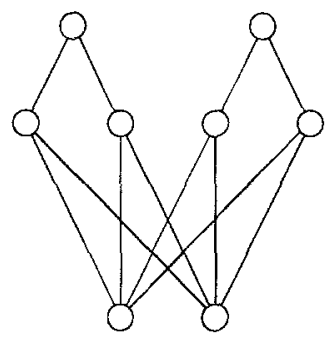

(c)

Figure 20 Minimal nonplanar castles.
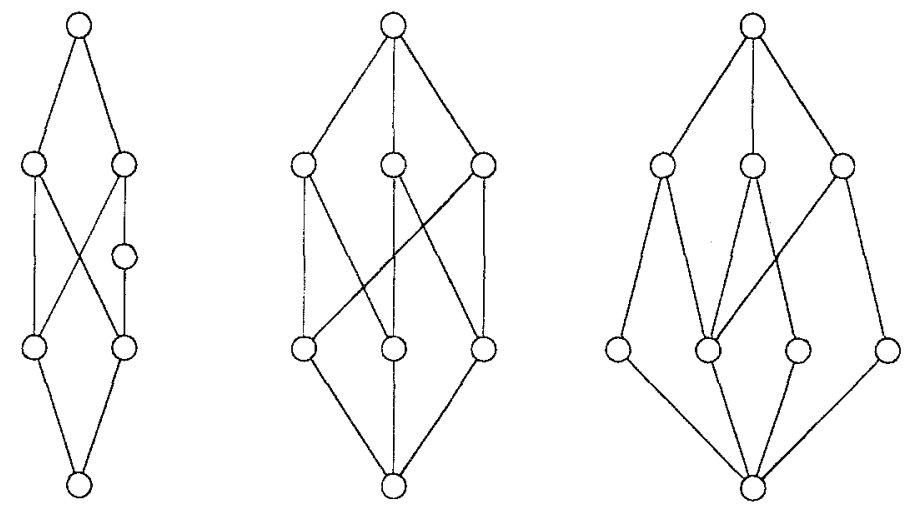

Figure 21 
dimension two? ( $k \geq 3$ because, page $(P)=3$ for the planar lattice $P$ in Figure 2). What about planar lattices?

3. Can we extend our result to (nonplanar) series-parallel ordered set?

What is an upper bound for the (nonplanar) series-parallel ordered set $P$, depending on the maximal $K_{m, n}$ 's in $P$.

For positive integers $m, n$ is there a function $f(m, n)$ such that for any series-parallel ordered set $P$

$\operatorname{page}(P) \leq \max \left\{f(m, n): K_{m, n}\right.$ is a maximal in $\left.P, m, n \geq 2\right\}$.

In particular, is there a positive integer $k$ such that

$f(m, n) \leq \min \{m, n\}+k$ for every maximal $K_{m, n}$ in $P$ ?

\section{References}

[1] F. Bernhart and P. C. Kainen (1979) The book thickness of a graph, Journal of Combinatorial Theory, Series B 27, 320-331.

[2] G. Birkhoff (1967) Lattice Theory, American Mathematical Society, Providence, Rhode Island.

[3] L. T. Q. Hung (1993) A Planar Poset which Requires four Pages, Ars Combin $35,291-302$

[4] H. de Fraysseix, P. O. de Mendez and J. Pach (1995) A Left Search Algorithm for Planar Graphs, Discrete Comput. Geom. 13, 459-468.

[5] D. Kelly and I. Rival (1975) Planar lattices, Canad. Journal of Mathematics $27,636-665$.

[6] H. M. MacNeille (1937) Partially ordered sets, Trans. Amer. Math. Soc. 42, 416-460.

[7] R. Nowakowski and A. Parker (1989), Ordered sets, pagenumbers and planarity, Order 6, 209-218.

[8] S. V. Pemmaraju (1992) Exploring the Powers of Stacks and Queues via Graph Layouts, Ph.D. thesis, Virginia Polytechnic Institute and State University at Blacksburg, Virginia.

[9] M. M. Systo (1990) Bounds to the Page Number of Partially Ordered Sets, Graph-theoretic concepts in computer science (Kerkrade, 1989),181-195, Lecture Notes in Comput. Sci. 411. Springer,Berlin.

[10] J. Valdes, R. E. Tarjan and E. L. Lawler (1982) The recognition of seriesparallel digraphs, SIAM J. Computing 11, 298-314.

[11] M. Yannakakis (1989) Embedding planar graphs in four pages, J. Comput. System Sci. 38, 36-67. 